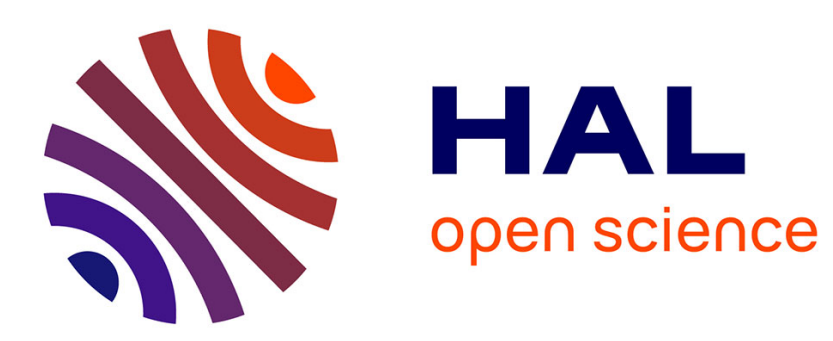

\title{
Multimodal vibration damping through a periodic array of piezoelectric patches connected to a passive network
}

Boris Lossouarn, Mathieu Aucejo, Jean-François Deü

\section{To cite this version:}

Boris Lossouarn, Mathieu Aucejo, Jean-François Deü. Multimodal vibration damping through a periodic array of piezoelectric patches connected to a passive network. SPIE Smart Structures and Materials + Nondestructive Evaluation and Health Monitoring, Apr 2015, San Diego, United States. $10.1117 / 12.2083835$. hal-01739217

\section{HAL Id: hal-01739217 \\ https://hal.science/hal-01739217}

Submitted on 21 Mar 2018

HAL is a multi-disciplinary open access archive for the deposit and dissemination of scientific research documents, whether they are published or not. The documents may come from teaching and research institutions in France or abroad, or from public or private research centers.
L'archive ouverte pluridisciplinaire HAL, est destinée au dépôt et à la diffusion de documents scientifiques de niveau recherche, publiés ou non, émanant des établissements d'enseignement et de recherche français ou étrangers, des laboratoires publics ou privés. 


\title{
Multimodal vibration damping through a periodic array of piezoelectric patches connected to a passive network
}

\author{
Boris Lossouarn $^{a}$, Mathieu Aucejo ${ }^{a}$ and Jean-François Dë̈ ${ }^{a}$ \\ ${ }^{a}$ Conservatoire National des Arts et Métiers, 2 Rue Conté, Paris, France
}

\begin{abstract}
In damping devices involving piezoelectric elements, a single piezoelectric patch cannot consistently achieve multimodal control because of charge cancellation or vibration node location. In order to sense and control structural vibration on a prescribed frequency range, a solution consists in using an array of several piezoelectric patches being small compared to the smallest wavelength to control. Then, as an extension of the tuned mass damper strategy, a passive multimodal control requires to implement a damping system whose modes are as close as possible to those of the controlled structure. In this way, the electrical equivalent of the discretized mechanical structure represents the passive network that optimizes the energy transfer between the two media. For onedimensional structures, a periodic distribution in several unit cells enables the use of the transfer matrix method applied on electromechanical state-vectors. The optimal passive networks are obtained for the propagation of longitudinal and transverse waves and a numerical implementation of the coupled behavior is performed. Compared to the more classical resonant shunts, the network topology induces promising multimodal damping and a reduction of the needed inductance. It is thus possible to create a completely passive electrical structure as it is demonstrated experimentally by using only purely passive components.
\end{abstract}

Keywords: vibration control, multimodal coupling, passive damping, piezoelectricity, shunt, electrical network

\section{INTRODUCTION}

In order to damp vibration of a structure with piezoelectric elements, various shunt strategies were proposed, as the tuning of resistive and resonant circuits $^{1-3}$ or the use of negative capacitance. ${ }^{4,5}$ Before choosing an optimal shunt, it is crucial to determine the geometry and the placement of the piezoelectric patch that avoid charge cancellation and vibration node locations. When focusing on multimodal control, those configurations can be difficult to avoid if using a single piezoelectric element. By distributing several piezoelectric patches all over the structure, it becomes yet possible to sense and control wavelength being large enough compared to the length of the patches. Periodic resonant distributions were investigated by Thorp et al. ${ }^{6}$ for longitudinal waves propagation and then by Airoldi and Ruzzene ${ }^{7,8}$ and Wang et al. ${ }^{9,10}$ for transverse waves propagation. Those studies considers independent shunts with no link between successive piezoelectric patches. A few papers deal with the possibility of connecting several patches together, as Maurini et al. ${ }^{11}$ or Bisegna et al. ${ }^{12}$ who analyzed the performance of several networks. Some networks induce interesting performance on a broad frequency range, when they approximate the dispersion relation of the mechanical waveguide. Indeed, as an extension of the tuned mass damper strategy, ${ }^{13}$ it is possible to reach a multimodal control by connecting a structure to its modal equivalent.

In this paper, a multimodal control is applied by using the electrical networks approximating the modal properties of a rod and a beam. In order to find those electrical equivalents, the mechanical media are discretized in lattices of point masses for longitudinal or transverse wave propagation. Then, an electromechanical analogy ${ }^{14,15}$ gives the network intended for being connected to the structure through piezoelectric patches. For a rod, it gives a line of inductors which is periodically connected to the ground through capacitors. ${ }^{16}$ For a beam, it is obtained

Further author information: (Send correspondence to B.L.)

B.L.: E-mail: boris.lossouarn@cnam.fr, Telephone: +1 4045198166

M.A.: E-mail: mathieu.aucejo@cnam.fr, Telephone: +33 (0)1 58808581

J.F.D.: E-mail: jean-francois.deu@cnam.fr, Telephone: +33 (0)1 40272760 
the same architecture as the network presented by Andreaus et al. ${ }^{17}$ which reveals transformers in addition to the inductors.

Concerning the analysis of the coupled problem, a periodic distribution of the piezoelectric patches enables to define a unit cell that can be considered as the building block of the layout. It becomes then possible to use a transfer matrix formulation. As presented by Lu and Tang ${ }^{18}$ for a similar problem, both the mechanical and the electrical variables are described into electromechanical state vectors that are defined at the ends of the unit cells. The transfer matrix is here obtained by considering a discrete model, which is based on global characteristics of the unit cell. The transfer matrix is then used to propagate any excitation all along the structure. It is thus possible to compute any frequency response function as well as global mechanical and electrical eigenmodes. In this way, the damping performances of the networks can be evaluated. It is seen that a multimodal network can match the resonances of a mechanical structure. The modal coupling conditions are given for both the rod and the beam. When the number of piezoelectric patches is increased, the network topology generates a decrease in the required inductance. Consequently, a resonant damping system can be developed without any synthetic inductors.

The multimodal damping strategy is validated by experiments that are conducted on a free-free structure covered with twenty pairs of piezoelectric patches. Both networks for damping of longitudinal and transverse waves are implemented with passive electrical components. It is observed significant vibration reductions simultaneously on the four first modes of the structures. A comparison with numerical computations based on the matrix transfer formulation is also presented. The results are sufficiently close to validate the model that it can then be used to characterize the influence of various network configurations.

\section{WAVE PROPAGATION IN ONE-DIMENSIONAL DISCRETE STRUCTURES}

A transfer matrix formulation involving force and velocity state vectors is introduced in order to model wave propagation in one-dimensional structures. Two lattices of point masses are then proposed as discrete representations of continuous media. The first lattice is dedicated to longitudinal wave propagation whereas the second describes transverse wave propagation. Both can be converted into their electrical analogues by applying the direct electromechanical analogy.

\subsection{Transfer Matrix Formulation}

A one-dimensional periodic structure is equivalent to a succession of identical unit cells. ${ }^{19}$ Each unit cell is described by a relation between the mechanical states at its right and left ends. This is expressed by a relation involving a transfer matrix $\boldsymbol{T}$ :

$$
\left[\begin{array}{c}
\dot{\boldsymbol{q}}_{\mathrm{R}} \\
\boldsymbol{F}_{\mathrm{R}}
\end{array}\right]=\boldsymbol{T}\left[\begin{array}{c}
\dot{\boldsymbol{q}}_{\mathrm{L}} \\
\boldsymbol{F}_{\mathrm{L}}
\end{array}\right],
$$

where $\boldsymbol{q}_{\mathrm{L}}$ and $\boldsymbol{q}_{\mathrm{R}}$ refer to the velocities at the left and right ends of the unit cell. $\boldsymbol{F}_{\mathrm{R}}$ is the force applied on the considered cell by its right neighbor and $\boldsymbol{F}_{\mathrm{L}}$ is the force applied by the considered unit cell on its left neighbor.

In a periodic structure, each unit cell is described by a same transfer matrix $\boldsymbol{T}$. Consequently, the mechanical state on the right of the $n^{\text {th }}$ unit cell is obtained by raising $\boldsymbol{T}$ to the power of $n$ :

$$
\left[\begin{array}{c}
\dot{q}_{n} \\
F_{n}
\end{array}\right]=T^{n}\left[\begin{array}{c}
\dot{q}_{0} \\
F_{0}
\end{array}\right]=\left[\begin{array}{cc}
T_{\dot{q} \dot{q}}^{n} & T_{\dot{q} F}^{n} \\
T_{F \dot{q}}^{n} & T_{F F}^{n}
\end{array}\right]\left[\begin{array}{c}
\dot{q}_{0} \\
F_{0}
\end{array}\right]
$$

where the subscript 0 represents the left end of the first unit cell. For a finite structure, the solution of the problem requires to consider the boundary conditions. For example, with a prescribed force $\boldsymbol{F}_{\mathbf{0}}$ applied to the left end of a free-free structure, the displacement $\boldsymbol{q}_{\boldsymbol{n}}$ at its right end is defined by:

$$
\dot{q}_{n}=\left(T_{\dot{q} F}^{n}-T_{\dot{q} \dot{q}}^{n} T_{F \dot{q}}^{n-1} T_{F F}^{n}\right) F_{0}
$$




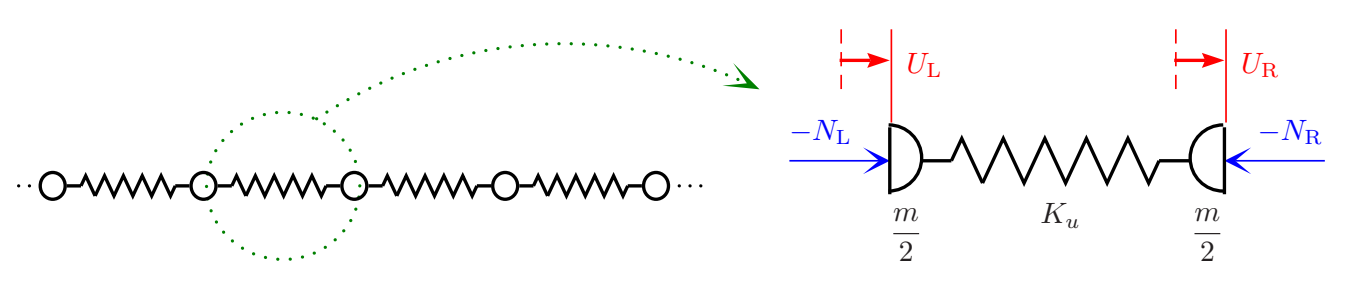

Figure 1. Discrete model for longitudinal wave propagation and the corresponding unit cell.

\subsection{Longitudinal Wave Propagation}

As introduced by Brillouin, longitudinal wave propagation can be analyzed with a periodic lattice model. ${ }^{16}$ If the considered wavelength is large enough compared to the length of the unit cells, a lattice of point masses turns out to be a suitable approximation of a continuous structure. This applies to a rod by considering periodically lumped masses $m$ linked serially with springs of stiffness $K_{u}$. The unit cell can then be described by two half masses at each ends of a spring, as shown in Fig. 1. The mass and the stiffness are determined from the local properties of the considered rod and the length $a$ of the unit cell: $m=\rho S a$ and $K_{u}=Y S / a$, where $\rho$ is the density of the rod, $Y$ its Young modulus and $S$ its cross-section.

The mechanical analysis of the unit cell presented in Fig. 1 gives two equations involving the longitudinal velocity $\dot{U}$ and the normal force $N$ at both left and right ends. The sign of the normal forces is assigned accordingly to the classical convention used in Eq. (1), which induces compression for negative forces. The two equations can be rearranged in the following transfer matrix form, $\omega$ being equal to the angular frequency:

$$
\left[\begin{array}{c}
\dot{U}_{\mathrm{R}} \\
N_{\mathrm{R}}
\end{array}\right]=\left[\begin{array}{cc}
1+f_{u} & \frac{j \omega}{K_{u}} \\
j\left(2+f_{u}\right) \frac{m}{2} \omega & 1+f_{u}
\end{array}\right]\left[\begin{array}{c}
\dot{U}_{\mathrm{L}} \\
N_{\mathrm{L}}
\end{array}\right], \quad \text { where } f_{u}=-\omega^{2} \frac{m}{2 K_{u}} \quad \text { and } \quad j=\sqrt{-1}
$$

With this transfer matrix and by applying Eq. (2) with $\dot{\boldsymbol{q}}=\dot{U}$ and $\boldsymbol{F}=N$, it thus becomes possible to analyze longitudinal wave propagation in a lattice with any number of unit cells and various boundary conditions.

\subsection{Transverse Wave Propagation}

As for the longitudinal wave propagation, a continuous structure subjected to transverse waves can be modeled by a lattice of point masses. This still requires to consider wavelength being large enough compared to the length of the unit cells. Under this condition, a beam can be discretized in a series of masses $m$ connected together by torsional springs of stiffness $K_{\theta}$. Those two constants come from the global properties of a beam portion of length $a$. Consequently, the mass $m$ is still equal to $\rho S a$ and $K_{\theta}=Y I / a$, where $I$ is the second moment of area of the beam's cross-section. A representation of a transverse lattice is proposed in Fig. 2, where each unit cell presents a bending stiffness which is serially divided in two torsional springs of stiffness $2 K_{\theta}$. For both left and right half masses, it is represented the transverse and angular displacements, $W$ and $\theta$, as well as the applied shear forces $Q$ and bending moments $M$.

The unit cell presented in Fig. 2 is analyzed for small displacement and no moment of inertia of the lumped masses. The two degrees of freedom of both half masses lead to four equations that can be expressed in a $4 \times 4$

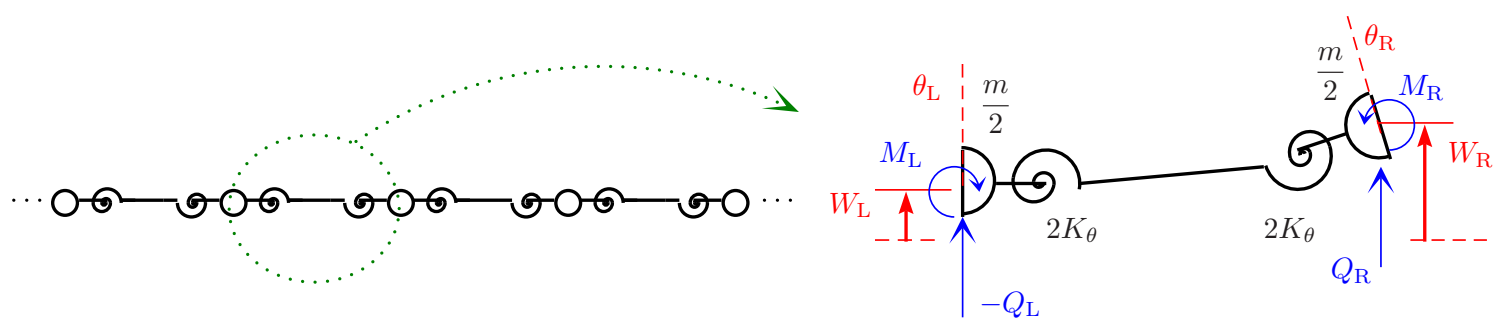

Figure 2. Discrete model for transverse wave propagation and the corresponding unit cell. 
transfer matrix:

$$
\left[\begin{array}{c}
\dot{W}_{\mathrm{R}} \\
\dot{\theta}_{\mathrm{R}} \\
M_{\mathrm{R}} \\
Q_{\mathrm{R}}
\end{array}\right]=\left[\begin{array}{cccc}
1 & a & \frac{j a \omega}{2 K_{\theta}} & 0 \\
-a f_{\theta} & 1 & \frac{j \omega}{K_{\theta}} & -\frac{j a \omega}{2 K_{\theta}} \\
-j a m \omega & 0 & 1 & -a \\
j 2 m \omega & j a m \omega & f_{\theta} & 1
\end{array}\right]\left[\begin{array}{c}
\dot{W}_{\mathrm{L}} \\
\dot{\theta}_{\mathrm{L}} \\
M_{\mathrm{L}} \\
Q_{\mathrm{L}}
\end{array}\right], \quad \text { where } f_{\theta}=-\omega^{2} \frac{m}{4 K_{\theta}}
$$

The matrix is here expressed for co-located springs and masses. This induces that the bar linking the two torsional spring of stiffness $2 K_{\theta}$ has the same length $a$ as the unit cell. Equation 2 can then be applied with $\dot{\boldsymbol{q}}=[\dot{W} ; \dot{\theta}]$ and $\boldsymbol{F}=[M ; Q]$ in order to obtain frequency response functions involving the state variables at the ends of the structure.

\subsection{Direct Electromechanical Analogy}

Both unit cells corresponding to longitudinal and transverse wave propagation can be transposed into electrical models by applying the direct electromechanical analogy. ${ }^{14,15}$ Referring to this analogy, a force is equivalent to a voltage and a velocity is equivalent to an intensity. Consequently, the analogue of a mass is an inductance and the analogue of a stiffness is the inverse of a capacitance. Then, the unit cell in Fig. 2 being made of two masses connected with a linear spring, its electrical analogue corresponds to a set of two inductors with a connections to the ground through a capacitor. This is illustrated in Fig. 3(a) where conventional electrical notations are replaced by their analogues that refer to the mechanical unit cell. It can be remarked that the analogues of the intensities are directly assigned to the previously defined velocities. With this choice, the two voltages are equivalent to the opposite of the normal forces in order to respect the sign convention.

The analogue of the mechanical unit cell presented in Fig. 2 is somewhat more complex as it requires to model a lever of length $a$. Indeed, for small displacements, this length $a$ refers to the proportionality constant between the transverse velocity difference $\dot{W}_{\mathrm{R}}-\dot{W}_{\mathrm{L}}$ and the angular velocity of the bar linking the two torsional springs. Moreover, $a$ is also the proportionality constant between the bending moment difference $M_{\mathrm{R}}-M_{\mathrm{L}}$ and the shear force in the same bar. This is modeled by an electrical transformer, where the ratio $a$ of the voltages on both windings is the inverse of the respective ratio of the intensities. It is thus obtained the electrical unit cell corresponding to transverse wave propagation as presented in Fig. 3(b), where torsional springs are also represented by capacitors and bending moments by voltages.

Periodic electrical networks are created by connecting identical electrical unit cells one after the other. The two networks based on the unit cells of Fig. 3 are the analogues of the lattices of point masses describing longitudinal and transverse wave propagation. At the end, the obtained longitudinal network corresponds to the one introduced by Brillouin ${ }^{16}$ as a low-pass electric filter . Regarding the transverse network, the same layout was obtained by Andreaus et al. ${ }^{17}$ after a discretization of the Euler beam equations.

(a)

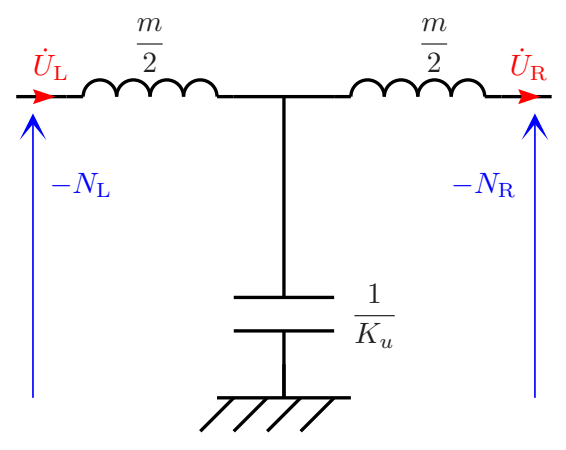

(b)

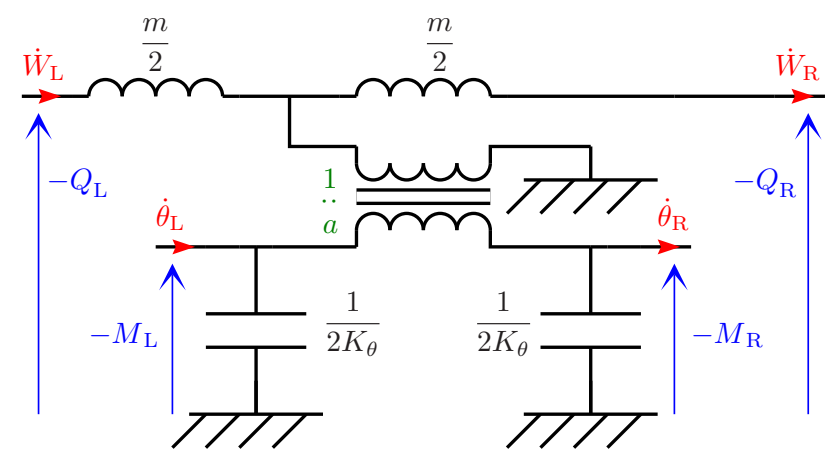

Figure 3. Electrical analogues of the mechanical unit cells: (a) Electrical unit cell corresponding to the longitudinal wave propagation. (b) Electrical unit cell corresponding to the transverse wave propagation. 


\section{MULTIMODAL COUPLING OF ONE-DIMENSIONAL STRUCTURES TO THEIR ELECTRICAL ANALOGUES}

In a goal of passive and broadband vibration attenuation, an array of piezoelectric patches is periodically distributed on a rod or a beam. It is seen that the corresponding electromechnical unit cells can be described by a discrete electrical model. This allows to determine the transfer matrix of a mechanical unit cell coupled to its analogous electrical circuit. Linking several unit cells in a row reveals a real electrical networks connecting all the piezoelectric patches. A tuning of the electrical components adjusts the electrical eigenmodes to those of the mechanical structure. It is thus obtained the equivalent of a multimodal tuned mass damper that can be implemented with limited values of inductance.

\subsection{Discrete Models for Electromechanical Unit Cells}

Previous analyses of one-dimensional structures covered by a periodic array of piezoelectric patches model the continuity of the mechanical medium. ${ }^{5-12,17,18,20,21}$ In this paper, it is chosen to replace the mechanical continuum by its discrete equivalent. This simplifies the problem and match the discrete nature of an electrical network. In order to find the discrete model of the considered electromechanical periodic structure, it is possible to firstly focus on the corresponding continuous unit cell. This one is represented in Fig. 4(a) for the case of longitudinal wave propagation. The rod portion have a length $a$, a width $b$ and a thickness $h_{\mathrm{s}}$. It is symmetrically covered with a pair of piezoelectric patches of length $l_{\mathrm{p}}$, width $b$ and thickness $h_{\mathrm{p}}$. The two patches are polarized in opposite directions and connected in parallel in order to act on longitudinal vibrations. ${ }^{6}$

The present purpose is then to transform the electromechanical unit cell into a discrete model based on global properties. From a purely mechanical point of view, a portion of rod is discretized as presented in Fig. 3(a). It still remains to model the piezoelectric coupling, which can be apprehended by using the model of a transformer, as illustrated in Fig. 4(b). The transformer converts a mechanical displacement into an electrical displacement and a voltage into an added force. This model, which comes from the linear piezoelectricity theory, is usually employed to describe a single piezoelectric patch. ${ }^{15}$ However, it is here extended to the entire unit cell. As for the discretization of a purely mechanical medium, this global model is only valid if the considered wavelength is long enough compared to the length of the patches.

Once the architecture of the discrete model is constituted, the global constants illustrated in Fig. 4(b) need to be defined. The mass $m$ involved in the mechanical lattice is still the total mass of the continuous unit cell. If $\rho_{\mathrm{s}}$ and $\rho_{\mathrm{p}}$ are the density of the rod structure and the density of the piezoelectric patches,

$$
m=\rho_{\mathrm{s}} S_{\mathrm{s}} a+2 \rho_{\mathrm{p}} S_{\mathrm{p}} l_{\mathrm{p}}, \quad \text { where } \quad S_{\mathrm{s}}=h_{\mathrm{s}} b \quad \text { and } \quad S_{\mathrm{p}}=h_{\mathrm{p}} b
$$

Then, it is seen from Fig 4(b) that the stiffness $K_{u}$ is the longitudinal stiffness of the unit cell when the piezoelectric patches are short circuited $\left(V_{u \mathrm{I}}=0\right)$. In this case, the equivalent Young modulus of the piezoelectric material is equal to $1 / s_{11}^{E}$, where $s_{11}^{E}$ is the elastic compliance at constant electric field. The Young modulus of

(a)

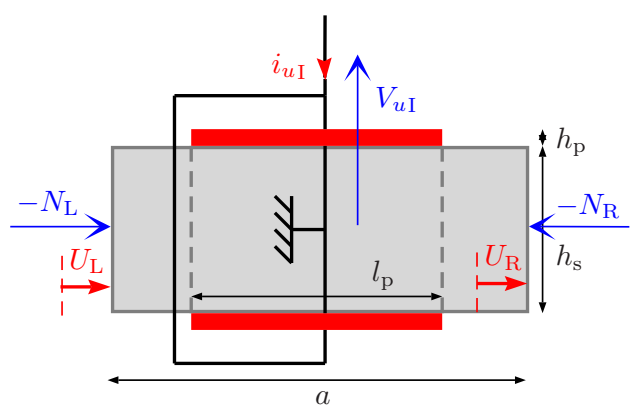

(b)

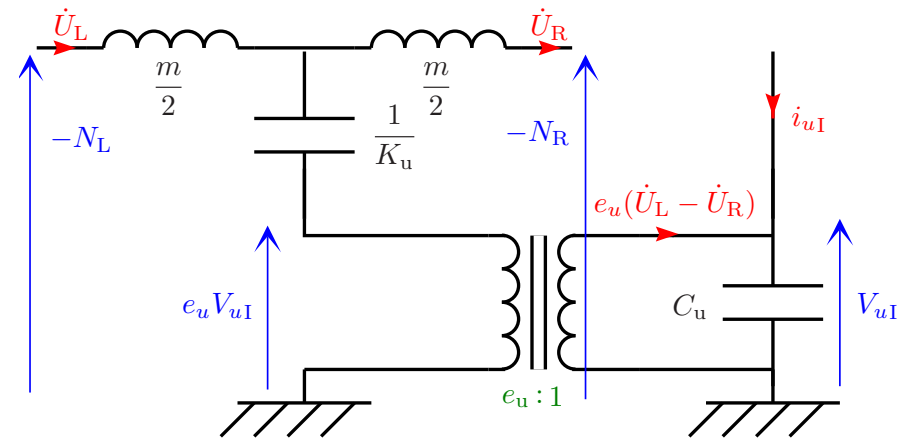

Figure 4. A unit cell involving piezoelectric patches: (a) Continuous model for the propagation of longitudinal waves. (b) Corresponding electrical model based on global properties. 
the rod being defined by $Y_{\mathrm{s}}$, the stiffness $K_{u}$ is obtained from the continuous unit cell when considering that all the cross-sections remain undeformed,

$$
\frac{1}{K_{u}}=\frac{l_{\mathrm{p}}}{Y_{\mathrm{s}} S_{\mathrm{s}}+2 Y_{\mathrm{p}}^{E} S_{\mathrm{p}}}+\frac{a-l_{\mathrm{p}}}{Y_{\mathrm{s}} S_{\mathrm{s}}}, \quad \text { where } \quad Y_{\mathrm{p}}^{E}=\frac{1}{s_{11}^{E}}
$$

The two remaining global constants are $C_{u}$ and $e_{u}$, the capacitance and the coupling coefficient of the whole unit cell. Fig 4(b) shows that $C_{u}$ corresponds to the capacitance obtained when $\dot{U}_{\mathrm{L}}-\dot{U}_{\mathrm{R}}=0$, namely for a constant relative position of the ends of the unit cell. This doesn't mean that the strain in the piezoelectric patch is constant as it can benefit from the elasticity of the material on which it is bounded. Consequently, the global capacitance is neither a blocked capacitance nor a free capacitance but something in between. Its determination requires to take into account 3D effect, as done by Maurini et al. ${ }^{22}$ This last reference focuses also on a global coupling coefficient comparable to $e_{u}$, which meets the same 3D influence. Another more practical solution is to experimentally measure the capacitance of the unit cell in a free configuration $\left(N_{L}=N_{R}=0\right)$. $C_{u}$ and $e_{u}$ are then determined from this measurement and the knowledge of piezoelectric material properties, as presented in a previous paper. ${ }^{23}$ At the end, all the global constants appearing in Fig. 4(b) are known and this electrical model can be used to approximate the behavior of a unit cell subjected to longitudinal wave propagation.

Concerning transverse wave propagation, the same methodology applies. The main difference lies in the fact that the mechanical part is no longer described by Fig. 3(a) but by Fig. 3(b). The mass $m$ of the unit cell is still the mass given in Eq. (6). For the bending stiffness $K_{\theta}$, it requires to consider $I_{\mathrm{s}}$ and $I_{\mathrm{p}}$ the second moments of area of the beam and patches cross-sections,

$$
\frac{1}{K_{\theta}}=\frac{l_{\mathrm{p}}}{Y_{\mathrm{s}} I_{\mathrm{s}}+2 Y_{\mathrm{p}}^{E} I_{\mathrm{p}}}+\frac{a-l_{\mathrm{p}}}{Y_{\mathrm{s}} I_{\mathrm{s}}}, \quad \text { where } \quad I_{\mathrm{s}}=b \frac{h_{\mathrm{s}}^{3}}{12} \quad \text { and } \quad I_{\mathrm{p}}=b \frac{\left(h_{\mathrm{s}}+2 h_{\mathrm{p}}\right)^{3}-h_{\mathrm{s}}^{3}}{24} .
$$

The global capacitance $C_{\theta}$ and the coupling coefficient $e_{\theta}$ related to transverse propagation are subjected to the same comments as their equivalents in longitudinal propagation. They can thus be obtained by $3 \mathrm{D}$ calculations or deduced from experimental measurements.

\subsection{Connection to the Analogous Networks}

The electromechanical unit cells were represented by discrete models when no electrical components are connected to the piezoelectric patches. As it was chosen to use electrical schemes, the addition of components simply requires to place them in the model with a connection to the piezoelectric capacitance. In this way, if identical inductors $L_{u}$ are connecting successive pairs of piezoelectric patches on a rod, the ensuing discrete unit cell is presented in Fig. 5. It can be remarked that the mechanical and electrical parts of the model have the same architecture. Therefore, inductors connecting successive patches give a network being the analogue a longitudinal lattice of point masses. All the "electrical stiffness" is supported by the capacitance of the piezoelectric patches. There is thus no need to add real capacitors to reach a lattice behavior.

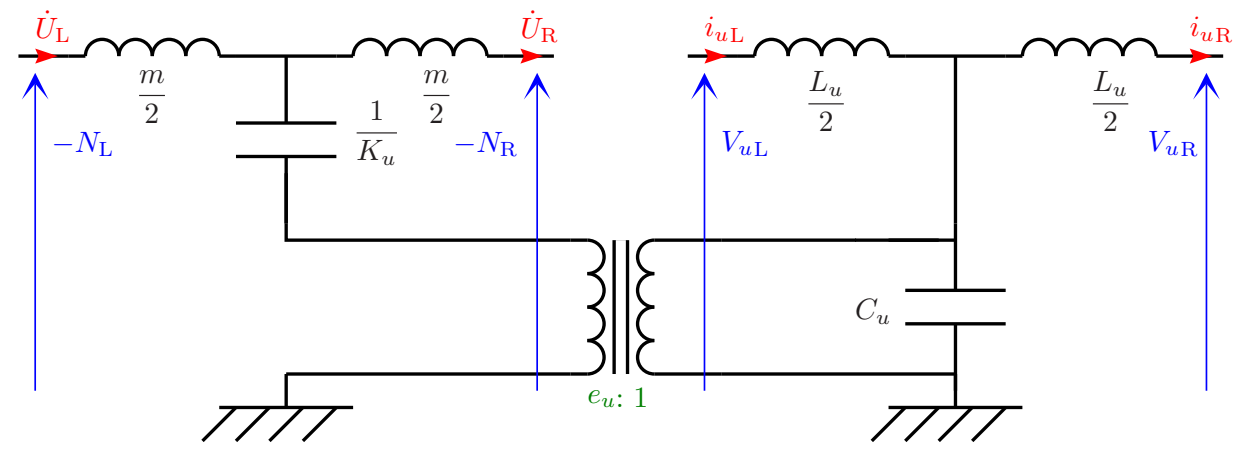

Figure 5. Electrical model of the longitudinal unit cell connected to its analogous network. 
The voltages $V_{u}$ and the currents $i_{u}$ are defined in Fig. 5 as the respective analogues of the normal forces $-N$ and the velocities $\dot{U}$. At each end, the state of the electromechanical unit cell is thus defined by two mechanical and two electrical variables. The unit cell can then be described by a $4 \times 4$ transfer matrix resulting from the electrical equations that governs the circuit in Fig. 5,

$$
\left[\begin{array}{c}
\dot{U}_{\mathrm{R}} \\
i_{u \mathrm{R}} \\
N_{\mathrm{R}} \\
V_{u \mathrm{R}}
\end{array}\right]=\left[\begin{array}{cccc}
1+f_{u} & -\frac{\Lambda_{u}}{e_{u}} \hat{f}_{u} & \frac{1}{Z_{\left\{1 / K_{u}\right\}}} & \frac{e_{u}}{Z_{\left\{1 / K_{u}\right\}}} \\
-e_{u} f_{u} & 1+\left(1+\Lambda_{u}\right) \hat{f}_{u} & -\frac{e_{u}}{Z_{\left\{1 / K_{u}\right\}}} & -\frac{\left(1+\Lambda_{u}\right)}{Z_{\left\{C_{u}\right\}}} \\
\left(2+f_{u}\right) Z_{\{m / 2\}} & -e_{u} f_{u} Z_{\left\{L_{u} / 2\right\}} & 1+f_{u} & e_{u} f_{u} \\
e_{u} f_{u} Z_{\left\{L_{u} / 2\right\}} & -\left(2+\left(1+\Lambda_{u}\right) \hat{f}_{u}\right) Z_{\left\{L_{u} / 2\right\}} & \frac{\Lambda_{u}}{e_{u}} \hat{f}_{u} & 1+\left(1+\Lambda_{u}\right) \hat{f}_{u}
\end{array}\right]\left[\begin{array}{c}
\dot{U}_{\mathrm{L}} \\
i_{u \mathrm{~L}} \\
N_{\mathrm{L}} \\
V_{u \mathrm{~L}}
\end{array}\right],
$$

where $Z_{\{m / 2\}}=j \frac{m}{2} \omega, Z_{\left\{1 / K_{u}\right\}}=\frac{K_{u}}{j \omega}, Z_{\left\{L_{u} / 2\right\}}=j \frac{L_{u}}{2} \omega, Z_{\left\{C_{u}\right\}}=\frac{1}{j C_{u} \omega}, f_{u}=\frac{Z_{\{m / 2\}}}{Z_{\left\{1 / K_{u}\right\}}}, \hat{f_{u}}=\frac{Z_{\left\{L_{u} / 2\right\}}}{Z_{\left\{C_{u}\right\}}}$ and $\Lambda_{u}=e_{u}^{2} \frac{Z_{\left\{C_{u}\right\}}}{Z_{\left\{1 / K_{u}\right\}}}$. The impedances $Z$ are obtained in agreement with the theoretical electrical components defined in Fig. 5. Yet, it is still possible to focus on more complex electrical models including internal resistance by adjusting the respective impedances. At last, it can be seen that $e_{u}=0$ decouples the electrical structure from the mechanical one. It is actually obtained by a superposition of two $2 \times 2$ transfer matrices on the form given in Eq. (4).

The electrical network approximating the beam dispersion relation requires to include transformers, as shown in Fig. 4(b). As for the longitudinal network, the piezoelectric patches assume the role of capacitors. Consequently, a transformer winding links two successive patches and the other winding is connected to a line of inductors, as seen in Fig. 6. With this choice of components and architecture, an electrical network is created that is the analogue of the transverse lattice presented in Fig. 2. The voltages $V_{w}$ and $V_{\theta}$ are the analogues of the shear forces $-Q$ and the bending moments $-M$. The currents $i_{w}$ and $i_{\theta}$ are the analogues of the velocities $\dot{W}$ and $\dot{\theta}$. All of this gives electromechanical state vectors made of 8 variables. Fig. 6 enable to define the corresponding $8 \times 8$ transfer matrix, which is given in Eq. (10). Again, $e_{\theta}=0$ decouples the two structures and it is found the same matrix elements as the ones presented in Eq. (5) together with their electrical equivalents.

At the end, the transfer matrices (9) and (10) can be used in Eq. (2) to analyze the coupling of a rod or a beam to their analogous network through an array of piezoelectric patches. Those two matrices are thus powerful tools to design electrical networks in a goal of controlling longitudinal and transverse vibration of one-dimensional structures.

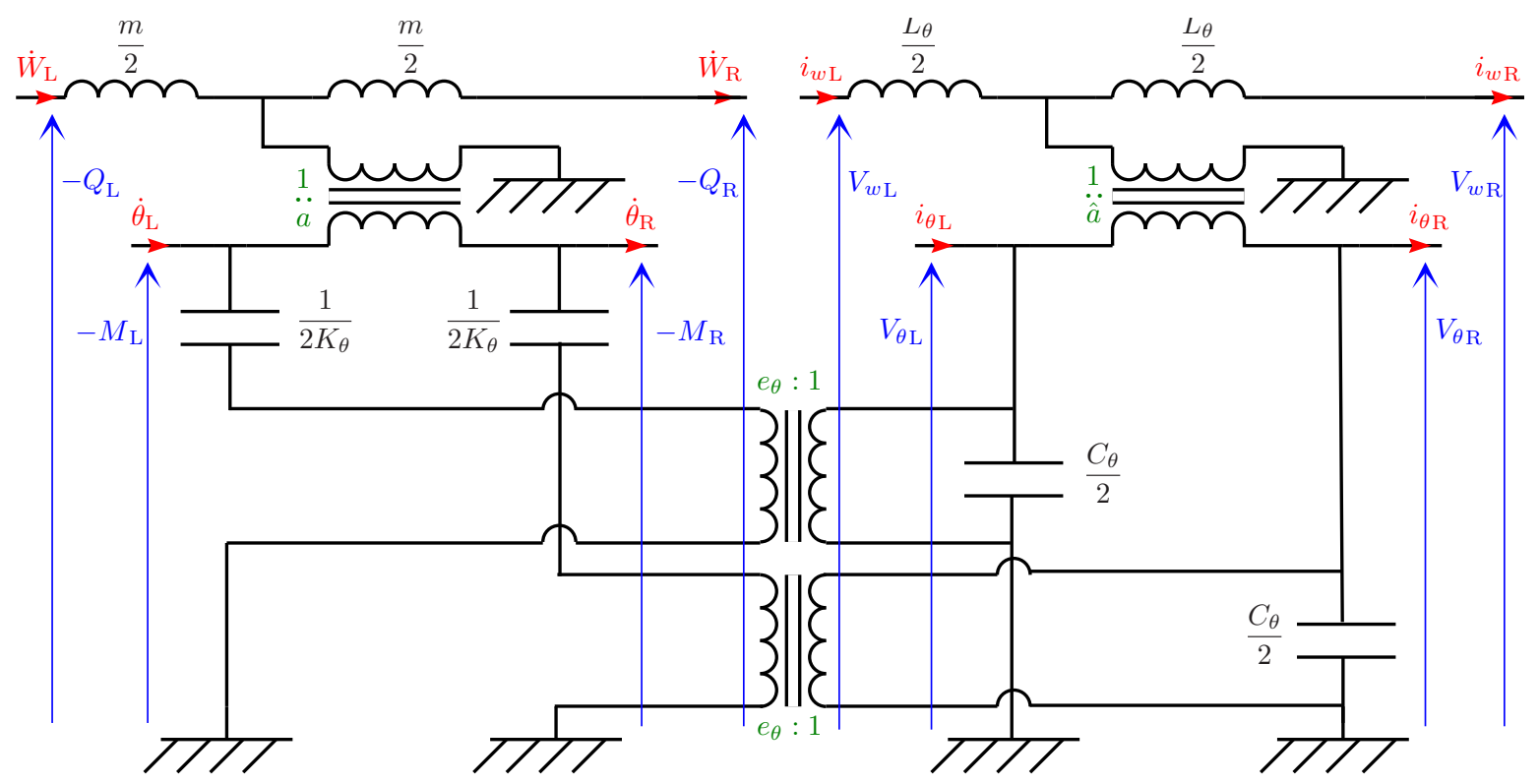

Figure 6. Electrical model of the transverse unit cell connected to its analogous network. 


$$
\left[\begin{array}{c}
\dot{W}_{\mathrm{R}} \\
\dot{\theta}_{\mathrm{R}} \\
i_{w \mathrm{R}} \\
i_{\theta \mathrm{R}} \\
M_{\mathrm{R}} \\
Q_{\mathrm{R}} \\
V_{\theta \mathrm{R}} \\
V_{w \mathrm{R}}
\end{array}\right]=\left[\begin{array}{cccccccc}
1 & a & 0 & 0 & \frac{a}{Z_{\left\{1 / 2 K_{\theta}\right\}}} & 0 & \frac{a e_{\theta}}{Z_{\left\{1 / 2 K_{\theta}\right\}}} & 0 \\
-a f_{\theta} & 1 & \frac{\hat{a} \Lambda_{\theta} \hat{f}_{\theta}}{e_{\theta}} & 0 & \frac{2}{Z_{\left\{1 / 2 K_{\theta}\right\}}}-\frac{a}{Z_{\left\{1 / 2 K_{\theta}\right\}}} & \frac{2 e_{\theta}}{Z_{\left\{1 / 2 K_{\theta}\right\}}} & -\frac{\hat{a} e_{\theta}}{Z_{\left\{1 / 2 K_{\theta}\right\}}} \\
0 & 0 & 1 & \hat{a} & -\frac{\hat{a} e_{\theta}}{Z_{\left\{1 / 2 K_{\theta}\right\}}} & 0 & -\frac{\hat{a}\left(1+\Lambda_{\theta}\right)}{Z_{\left\{C_{\theta} / 2\right\}}} & 0 \\
a e_{\theta} f_{\theta} & 0 & -\hat{a}\left(1+\Lambda_{\theta}\right) \hat{f}_{\theta} & 1 & -\frac{2 e_{\theta}}{Z_{\left\{1 / 2 K_{\theta}\right\}}} & \frac{a e_{\theta}}{Z_{\left\{1 / 2 K_{\theta}\right\}}} & -\frac{2\left(1+\Lambda_{\theta}\right)}{Z_{\left\{C_{\theta} / 2\right\}}} & \frac{\hat{a}\left(1+\Lambda_{\theta}\right)}{Z_{\left\{C_{\theta} / 2\right\}}} \\
-a Z_{\{m / 2\}} & 0 & 0 & 0 & 1 & -a & 0 & 0 \\
2 Z_{\{m / 2\}} & a Z_{\{m / 2\}} & 0 & 0 & a f_{\theta} & 1 & a e_{\theta} f_{\theta} & 0 \\
0 & 0 & \hat{a} Z_{\left\{L_{\theta} / 2\right\}} & 0 & 0 & 0 & 1 & -\hat{a} \\
0 & 0 & -2 Z_{\left\{L_{\theta} / 2\right\}} & -\hat{a} Z_{\left\{L_{\theta} / 2\right\}} & \frac{\hat{a} \Lambda_{\theta} \hat{f}_{\theta}}{e_{\theta}} & 0 & \hat{a}\left(1+\Lambda_{\theta}\right) \hat{f}_{\theta} & 1
\end{array}\right]\left[\begin{array}{c}
\dot{W}_{\mathrm{L}} \\
\dot{\theta}_{\mathrm{L}} \\
i_{w \mathrm{~L}} \\
i_{\theta \mathrm{L}} \\
M_{\mathrm{L}} \\
Q_{\mathrm{L}} \\
V_{\theta \mathrm{L}} \\
V_{w \mathrm{~L}}
\end{array}\right]
$$

where $Z_{\{m / 2\}}=j \frac{m}{2} \omega, Z_{\left\{1 / 2 K_{\theta}\right\}}=\frac{2 K_{\theta}}{j \omega}, Z_{\left\{L_{\theta} / 2\right\}}=j \frac{L_{\theta}}{2} \omega, Z_{\left\{C_{\theta} / 2\right\}}=\frac{2}{j C_{\theta} \omega}, f_{\theta}=\frac{Z_{\{m / 2\}}}{Z_{\left\{1 / 2 K_{\theta}\right\}}}, \hat{f}_{\theta}=\frac{Z_{\left\{L_{\theta} / 2\right\}}}{Z_{\left\{C_{\theta} / 2\right\}}}$ and $\Lambda_{u}=e_{\theta}^{2} \frac{Z_{\left\{C_{\theta} / 2\right\}}}{Z_{\left\{1 / 2 K_{\theta}\right\}}}$

\subsection{Modal Coupling Conditions}

Referring to the longitudinal unit cell in Fig. 1, another unit cell having the same layout would present equivalent modal properties if it has the same ratio $K_{u} / m$. By extension, two longitudinal lattices having identical boundary conditions and a same ratio $K_{u} / m$ present the same natural frequencies and the same mode shapes. Those conclusions arise also with the transverse lattice of Fig. 2 when the ratio $K_{\theta} /\left(a^{2} m\right)$ is kept the same. Consequently, the electromechanical analogy gives the modal coupling conditions that are used to tune the networks illustrated in Figs. 5 and 6:

$$
\frac{1}{L_{u} C_{u}}=\frac{K_{u}}{m} \text { and } \quad \frac{1}{\hat{a}^{2}} \frac{1}{L_{\theta} C_{\theta}}=\frac{1}{a^{2}} \frac{K_{\theta}}{m} .
$$

By applying those coupling conditions, the electrical networks hold the same dispersion relations as their mechanical counterpart. Adding analogous boundary conditions leads to identical modal properties. As energy can flows through the piezoelectric patches, it creates the equivalent of a multimodal tuned mass damper. ${ }^{13}$ This represents the extension of previous works involving passive resonant shunts that are tuned independently to a single natural frequency. ${ }^{6,8,9}$ Here is added a space dimension because the resonances don't only match along the frequency domain but also on the spatial domain thanks to similar mode shapes. When considering the continuous mechanical structures, it is remarked that their modal properties differ from those of discrete lattices. Consequently, the natural frequencies of the rod and the beam can't match exactly those of the corresponding network. However, this difference becomes negligible with a sufficiently high number of unit cell per wavelength. It is thus essential to determine the highest mode to be controlled before choosing the number of piezoelectric patches that will cover the mechanical structure.

The global capacitance of a unit cell being approximately proportional the surface area of the piezoelectric patches, it can be considered proportional to $1 / n$, where $n$ is the number of unit cells for a fixed thickness of the patches and fixed length and width of the main structure. So, with a prescribed amount of piezoelectric material, it is seen from the modal coupling conditions (11) and the definition of the global constants that $L_{u} \propto 1 / n$ and $L_{\theta} \propto 1 / n^{3}$. Those conclusions were highlighted by Maurini et al. ${ }^{11}$ from the analysis of an electrical continuum. The proportionality relations are here illustrated from the discrete formulation but still implies that an increase in the number of unit cells leads to lower values of the required inductance. As a consequence, it becomes possible to consider low frequency applications without being limited by available values of passive inductors.

\section{EXPERIMENTAL RESULTS}

Twenty pairs of piezoelectric patches are periodically distributed on an aluminum bar. Both networks for longitudinal and transverse control are successively implemented with passive electrical components. Broadband vibration reduction is then observed with the two networks as expected by the discrete model based on transfer matrix formulation. 
Table 1. Geometry and material properties.

\begin{tabular}{|l|l|l|}
\hline & Rod (Aluminum 2017) & Patches (PZT) \\
\hline Length & $l_{\mathrm{s}}=n a=20 \times 5 \mathrm{~cm}$ & $l_{\mathrm{p}}=3 \mathrm{~cm}$ \\
\hline Width & $b=2 \mathrm{~cm}$ & $b=2 \mathrm{~cm}$ \\
\hline Thickness & $h_{\mathrm{s}}=2 \mathrm{~cm}$ & $h_{\mathrm{p}}=0.5 \mathrm{~mm}$ \\
\hline Density & $\rho_{\mathrm{s}}=2780 \mathrm{~kg} / \mathrm{m}^{3}$ & $\rho_{\mathrm{p}}=7800 \mathrm{~kg} / \mathrm{m}^{3}$ \\
\hline Young modulus & $Y_{\mathrm{s}}=73.9 \mathrm{GPa}$ & $1 / s_{11}^{E}=66.7 \mathrm{GPa}$ \\
\hline Charge constant & - & $d_{31}=-210 \mathrm{pC} / \mathrm{N}$ \\
\hline Permittivity & - & $\epsilon_{33}^{\sigma}=21.2 \mathrm{nF} / \mathrm{m}$ \\
\hline
\end{tabular}

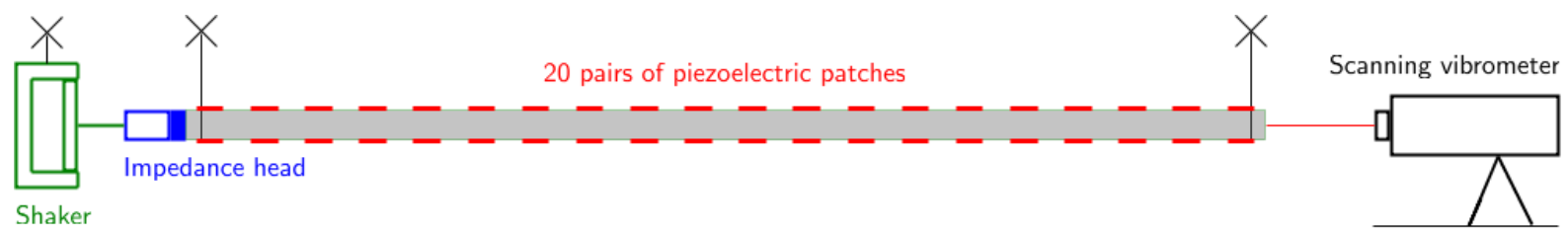

Figure 7. Experimental setup for the analysis of longitudinal waves.

\subsection{Experimental setup}

The main structure is a one meter aluminum bar, which is periodically covered with $n=20$ pairs of piezoelectric patches. Referring to Fig. 4(a), the geometry of the setup is presented in Table 1. This table described also the properties of the selected piezoelectric ceramic. The piezoelectric patches are linked together with a set of passive electrical components. It is either a line of inductors for longitudinal control or the network involving transformers and inductors for transverse control. Since the passive components have inherent internal resistance, there is no need to add resistors in the network. The tuning is validated by applying a voltage at one end of the network and measuring the voltage in the middle. This give electrical frequency response functions where appear the electrical resonances.

Once tuned, the resulting electromechanical structure is suspended by elastic straps in order to approximate free-free boundary conditions. A suspended shaker is connected to one end of the bar through an impedance head that measures the acceleration and the transmitted force. A scanning laser vibrometer measures the velocity at the other end. Figure 7 shows the setup and Fig. 8(a) a detail of the impedance head for the analysis of longitudinal waves. For transverse wave propagation, all the material remains the same but the shaker and the vibrometer are placed perpendicularly to the bar. A white noise excitation is generated and two signals are analyzed: the velocity measured by the vibrometer and the force measured by the impedance head. This gives the frequency response function defined by the velocity at the free end on the force at the excited end.

(a)

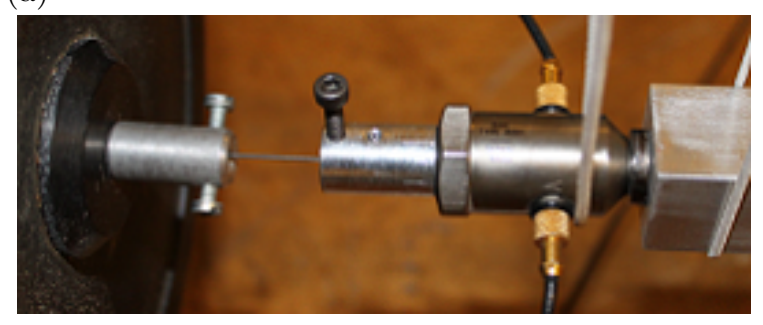

(b)

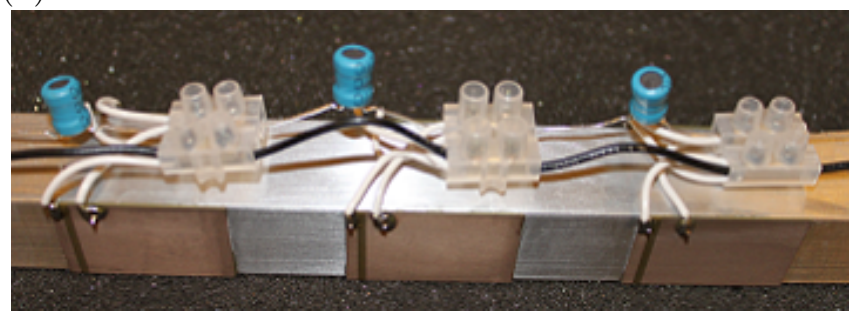

Figure 8. Details of the experimental setup for the damping of longitudinal waves: (a) Placement of the impedance head. (b) Electrical network involving inductors. 


\subsection{Damping of Longitudinal Waves}

The first experiment corresponds to the validation of the multimodal damping strategy for longitudinal propagation. The capacitance $C_{u}$ being evaluated to $36 \mathrm{nF}$, the modal coupling condition (11) gives an inductance $L_{u}$ equal to $2.8 \mathrm{mH}$. The electrical network was thus realized by linking successive unit cells with standard $2.8 \mathrm{mH}$ inductors, as seen in Fig. 8(b). The ends of the network are both closed by a $L_{u} / 2$ inductance. This comes from the unit cell architecture and the fact that a free mechanical boundary condition is equivalent to a zero voltage.

The tuning of the electrical network is verified before looking at its effect on vibration reduction. A white noise voltage is applied at one end of the network and the voltage $V_{u}$ is measured in the center of the line of inductors. The electrical frequency response function presented in Fig. 9(a) is obtained. By analogy with the mechanical structure, this situation is equivalent to applying a force at one end of the rod and measuring the force in the middle of the rod. As a free-free configuration is considered, the middle of the rod is a node for the even force modes. The same remark thus applies to the voltage. This is confirmed by the electrical frequency response function where only the odd resonances appears. Then, it is seen sharp antiresonances centered on the electrical resonances. This validates the tuning of the network as the electrical antiresonances are related to the mechanical resonances. Indeed, energy injected in the electrical network is transferred to the rod by excitation of its eigenmodes through the piezoelectric patches. This creates antiresonances in the electrical spectrum.

The $4 \times 4$ matrix defined in (9) is used to compare the experimental results and the model developed in Sec. 3 . For a broadband analysis, it was observed that both serial and parallel internal resistance need to be included in the model. Those values were obtained by direct measurements on the electrical components. At the end, Fig. 9(a) shows a good agreement between the numerical and the experimental results. It can still be observed that the numerical antiresonances are deeper than the experimental ones. This can be explained by the fact that no mechanical damping was introduced in the model. The mechanical damping evaluated experimentally being considerably lower than the electrical damping, it has a negligible influence on the response once the network is tuned. Moreover, it is seen that the second numerical antiresonance is placed at a slightly lower frequency than the experimental one. This is due to the discrete approximation made in the model. Even if it remains limited for the first modes, it is important to remember that this error becomes non-negligible at higher frequencies.

(a)

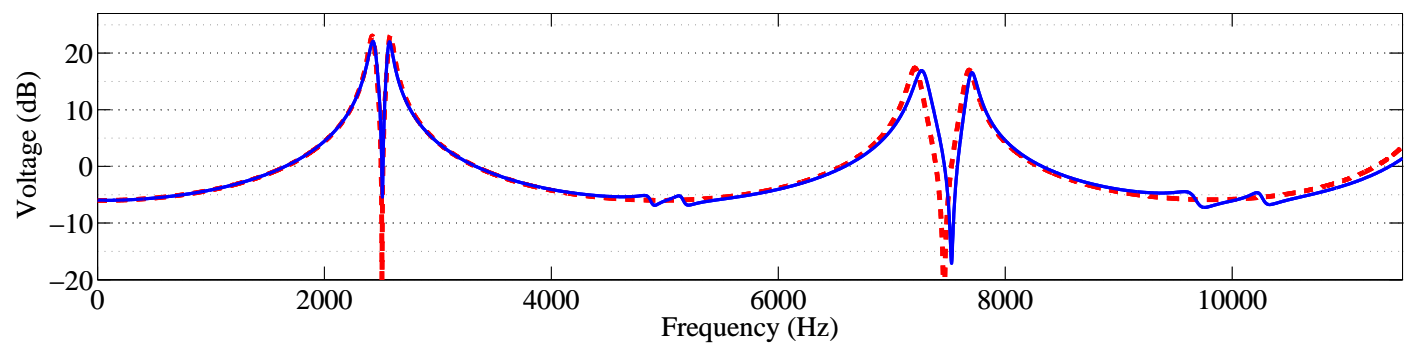

(b)

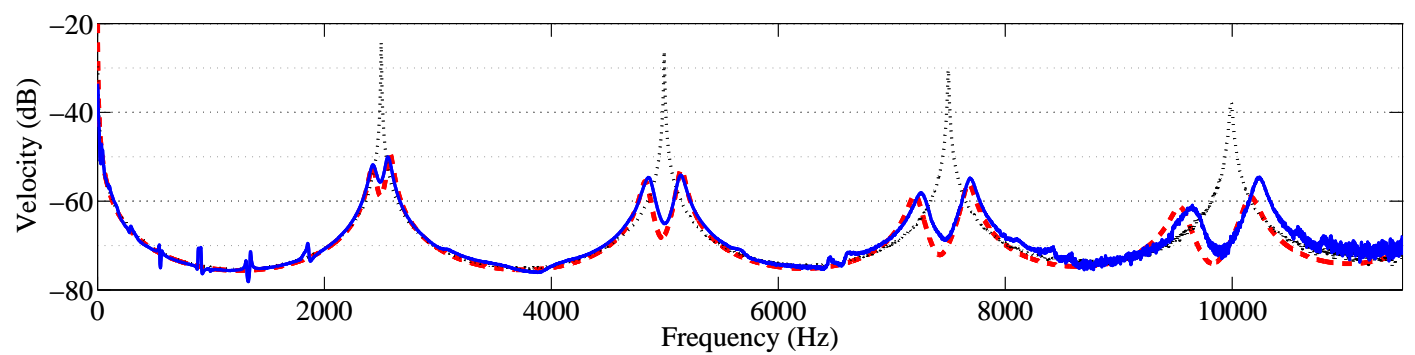

Figure 9. Multimodal damping of longitudinal waves: (a) Electrical frequency response functions - (-) for the experiment with a tuned network, (--) for the transfer matrix model with a tuned network. (b) Mechanical frequency response functions $-(\cdots)$ for the experiment without network, $(-)$ for the experiment with a tuned network and (--) for the transfer matrix model with a tuned network. 
Once the tuning of the electrical network is validated, the performance of the multimodal coupling can be evaluated. Figure 9(b) represents experimental frequency response functions, firstly without network and then with a tuned network. The performance is significant as the reduction is close to $25 \mathrm{~dB}$ for the first three resonances and close to $20 \mathrm{~dB}$ for the fourth one. This highlights the interest of the multimodal damping through an electrical network, which shows an efficient vibration control on a broad frequency range. Moreover, the model based on the transfer matrix formulation gives a suitable approximation of the vibration reduction.

The multimodal control strategy is efficient as long as the length of the unit cells is small compared to the smallest wavelength to control. Otherwise, the discrete approximation is no longer valid and the resonances of the networks move away from the resonances of the continuous structure. This is observed on the fourth mode where a shift is pronounced. This is due to the position of the fourth electrical resonance, which is lower than the corresponding mechanical resonance. This effect also explains some differences between the numerical results and the experiments. As the model is based on a full discretization of both the electrical and mechanical media, it can not take into account the mistuning between the actual resonances. This mistuning remains limited until the fourth mode but would becomes non-negligible for higher mode numbers. If more modes needs to be controlled, it is still possible to increase the number of unit cells. This simply enhances the frequency range in which the discrete network approximates a continuous structure.

\subsection{Damping of Transverse Waves}

The second experimental validation concerns the damping of transverse waves with the analogous network of a beam. The shaker and the vibrometer are placed perpendicularly to the beam at opposite ends, as seen in Fig. 10(a). The network described in Fig. 6 is implemented by using transformers with two windings in their secondary. Each winding is connected to one of the two patches of a unit cell. The transformers, as well as the inductors don't come from standard series but were manufactured in order to obtain sufficiently low internal resistance. Those magnetic components were created by winding copper wire around ferrite cores. By taking into account the influence of the capacitance between windings it is obtained a global capacitance $C_{\theta}$ equal to $60 \mathrm{nF}$. Then, as the equivalent transformer ratio $\hat{a}$ is set to one, the modal coupling condition (11) shows that the inductors need to be close to $L_{\theta}=120 \mathrm{mH}$. All of this gives the network presented in Fig. 10(b), where the components on the bottom of the picture represent the line of inductors and the ones just above are the transformers.

As for the longitudinal damping, the tuning of the transverse network is controlled by looking at it electrical response. A white noise voltage is applied to one end of the network and the voltage $V_{w}$ is measured in the middle. The corresponding frequency response function is represented in Fig. 11(a), where appear together the experimental result and the numerical computation based on the transfer matrix model without mechanical damping. It is verified that electrical resonances are centered on sharp antiresonances, which correspond to

(a)

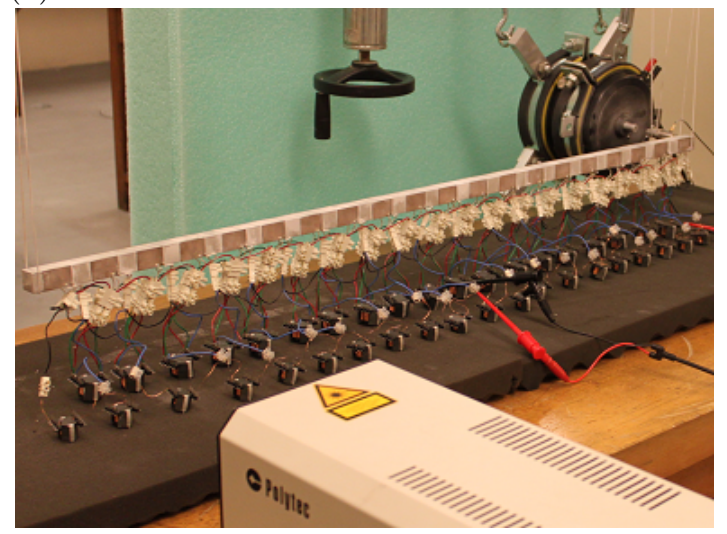

(b)

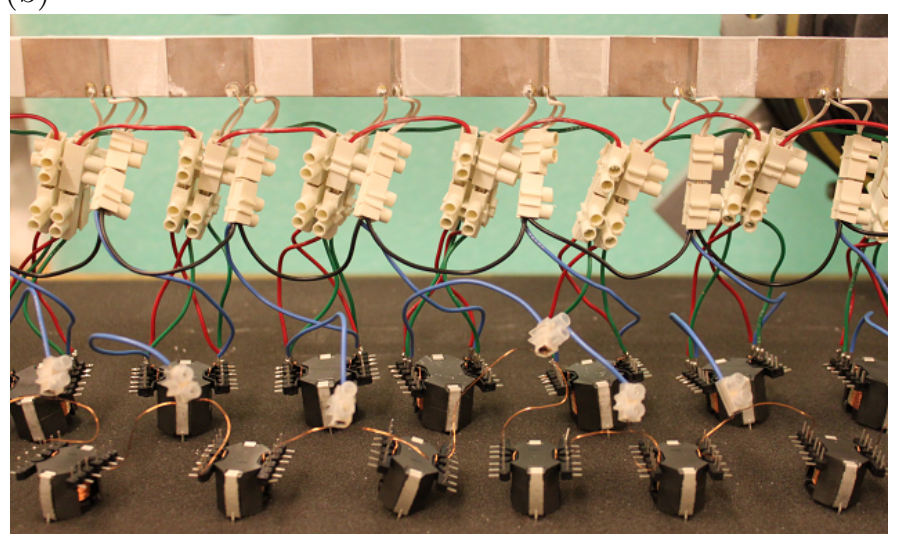

Figure 10. Details of the experimental setup for the damping of transverse waves: (a) Placement of the shaker and the scanning vibrometer. (b) Electrical network involving inductors and transformers. 
(a)

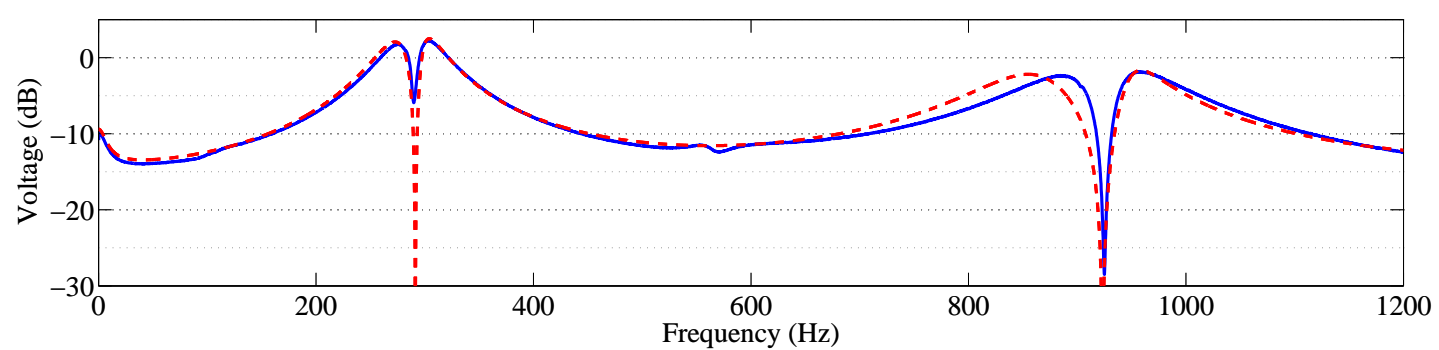

(b)

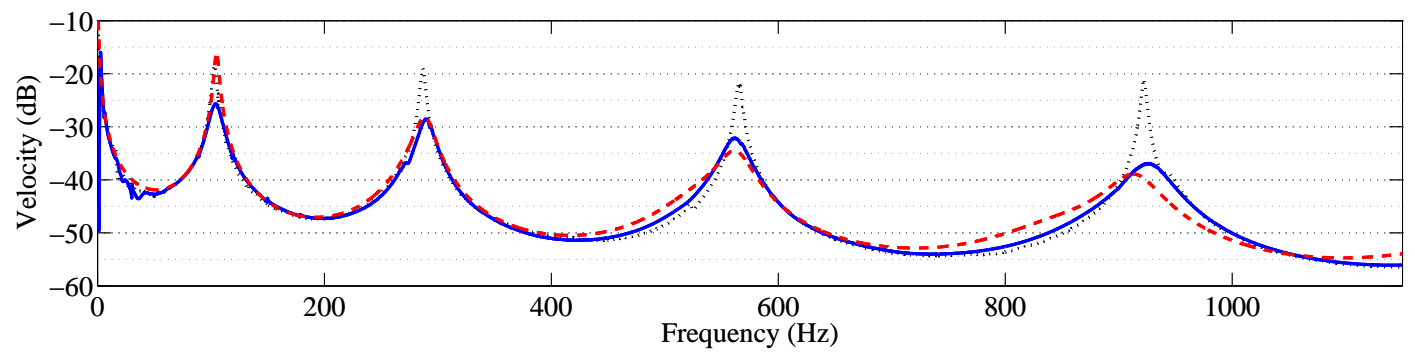

Figure 11. Multimodal damping of transverse waves: (a) Electrical frequency response functions - $(-)$ for the experiment with a tuned network, (--) for the transfer matrix model with a tuned network. (b) Mechanical frequency response functions $-(\cdots)$ for the experiment without network, $(-)$ for the experiment with a tuned network and (--) for the transfer matrix model with a tuned network.

transverse mechanical resonances. However, for bending modes, the middle position of a free-free beam is a node for the odd shear force modes. As a consequence, the measured voltage tracks only the even modes. The lowest frequency resonance observable in the response is thus the second electrical resonance of the network. The first one is placed around $100 \mathrm{~Hz}$, which corresponds to the first mode of the beam.

The effect of the network on structural vibrations is illustrated in Fig. 11(b). The transfer matrix model gives results that are close to the experiments for the modes 2, 3 and 4 but not for the first mode. This can be explained by the fact that the experimental setup adds a non-negligible mechanical damping through the wires and the connectors of the network. This mainly affects the first mode, which presents the largest displacement amplitude in the experiments. Nevertheless, the strategy is validated for the other modes, where it is observed reductions around $10 \mathrm{~dB}$ for the second and third modes and $15 \mathrm{~dB}$ for the fourth mode. The model predicts slightly larger reductions as it doesn't take into account the mistuning between the mechanical and the electrical resonances. At the end, it can still be remarked that the system is overdamped, no double peaks appear on both sides of the initial mechanical resonances. This is due to the internal resistance of the electrical components which is still too high for the present experiment. The reduction of the internal resistance is thus a key point that need to be solved to obtain better performance and to be able to look at low frequency applications.

\section{CONCLUSIONS}

A passive and broadband piezoelectric control is implemented for the damping of one-dimensional waves. First, longitudinal and transverse lattices are described along with a transfer matrix formulation that enables the analysis of periodic structures. Then, the electrical analogues of the lattices are given. They represent the electrical networks that can approximate the modal behavior of a rod or a beam. When considering that those structure are periodically covered with piezoelectric patches, it is possible to focus on a single unit cell. After dicretizing the mechanical medium, the electromechanical unit cell can be described by an electrical model based on global constants. Any electrical network linking the piezoelectric patches can be modeled by adding electrical components in the unit cell. This is here performed for the analogous networks of the longitudinal and transverse lattices. The model of the unit cell is then used to define the transfer matrix, which takes into 
account the coupling between the electrical and mechanical variables. Consequently, a model is obtained that allows to analyze various tuning configurations and their effect on vibration reduction. The proposed modal coupling conditions approximates the modal properties of the rod and the beam. It is thus obtained networks that have the same natural frequencies and mode shapes as the structures on which they are connected. This creates a distributed multimodal coupling being equivalent to a tuned mass effect occurring simultaneously on several modes.

The multimodal control strategy is experimentally validated for the damping of a rod and a beam. The corresponding networks are realized by involving the capacitance of the piezoelectric patches and magnetic components such as inductors and transformers. Without any external power, significant reductions is observed over a broad frequency range. Moreover, the transfer matrix model gives a suitable approximation of the electromechanical behavior, as long as the considered wavelength is large compared to the unit cells. Finally, a completely passive control solution is proposed with only the use of passive electrical components. This is made possible by the network topology that reduces the value of the required inductors. Therefore, issues are not related to the inductance level but to the procurement of passive components with a sufficiently low internal resistance.

\section{ACKNOWLEDGMENTS}

This work was funded by the French Ministry of National Education, Higher Education and Research through a three year scholarship for doctoral studies related to structural damping with piezoelectric devices. Thanks are also given to the Fulbright Program which makes possible a nine-month research visit at the Georgia Institute of Technology.

\section{REFERENCES}

[1] N. W. Hagood and A. von Flotow, "Damping of structural vibrations with piezoelectric materials and passive electrical networks," J. Sound Vib. 146, pp. 243-268, 1991.

[2] O. Thomas, J.-F. Deü, and J. Ducarne, "Vibrations of an elastic structure with shunted piezoelectric patches: efficient finite element formulation and electromechanical coupling coefficients," Int. J. Numer. Meth. Eng. 80, pp. 235-268, 2009.

[3] O. Thomas, J. Ducarne, and J.-F. Deü, "Performance of piezoelectric shunts for vibration reduction," Smart Mater. Struct. 21, p. 015008, 2012.

[4] B. S. Beck, K. A. Cunefare, and M. Collet, "Experimental assessment of negative impedance shunts for vibration suppression on a beam," in The 15th International Symposium on: Smart Structures and Materials 8 Nondestructive Evaluation and Health Monitoring, I. S. for Optics and Photonics, eds., Proc. SPIE 6928, pp. 69281X-69281X-9, 2008.

[5] B. S. Beck, K. A. Cunefare, M. Ruzzene, and M. Collet, "Experimental analysis of a cantilever beam with a shunted piezoelectric periodic array," J. Int. Mater. Sys. Struct. 22, pp. 1177-1187, 2011.

[6] O. Thorp, M. Ruzzene, and A. Baz, "Attenuation and localization of wave propagation in rods with periodic shunted piezoelectric patches," Smart Mater. Struct. 10, p. 979, 2001.

[7] L. Airoldi and M. Ruzzene, "Design of tunable acoustic metamaterials through periodic arrays of resonant shunted piezos," New J. Phys. 13, p. 113010, 2011.

[8] L. Airoldi and M. Ruzzene, "Wave propagation control in beams through periodic multi-branch shunts," $J$. Int. Mater. Sys. Struct. 22, pp. 1567-1579, 2011.

[9] G. Wang, S. Chen, and J. Wen, "Low-frequency locally resonant band gaps induced by arrays of resonant shunts with antoniou's circuit: experimental investigation on beams," Smart Mater. Struct. 20, p. 015026, 2011.

[10] G. Wang, J. Wang, S. Chen, and J. Wen, "Vibration attenuations induced by periodic arrays of piezoelectric patches connected by enhanced resonant shunting circuits," Smart Mater. Struct. 20, p. 125019, 2011.

[11] C. Maurini, F. dell'Isola, and D. Del Vescovo, "Comparison of piezoelectronic networks acting as distributed vibration absorbers," Mech. Syst. Signal Process. 18, pp. 1243-1271, 2004. 
[12] P. Bisegna, G. Caroso, and F. Maceri, "Optimized electric networks for vibration damping of piezoactuated beams," J. Sound Vib.. 289, pp. 908-937, 2006.

[13] J. P. Den Hartog, Mechanical vibrations, McGraw-Hill, New York, 1940.

[14] A. Bloch, "Electromechanical analogies and their use for the analysis of mechanical and electromechanical systems," J Inst. Electr. Eng. 92, pp. 157-169, 1945.

[15] L. L. Beranek, Acoustics, McGraw-Hill, New York, 1954.

[16] L. Brillouin, Wave propagation in periodic structures, McGraw-Hill, New York, 1946.

[17] U. Andreaus, F. dell'Isola, and M. Porfiri, "Piezoelectric passive distributed controllers for beam flexural vibrations," J. Vib. Contr. 10, pp. 625-659, 2004.

[18] Y. Lu and J. Tang, "Electromechanical tailoring of structure with periodic piezoelectric circuitry," J. Sound Vib.. 331, pp. 3371-3385, 2012.

[19] D. M. Mead, "Wave propagation in continuous periodic structures: research contribution from southampton," J. Sound Vib. 190, pp. 495-524, 1996.

[20] S. Vidoli and F. dell'Isola, "Modal coupling in one-dimensional electromechanical structured continua," Acta Mech. 141, pp. 37-50, 2000.

[21] F. dell'Isola, C. Maurini, and M. Porfiri, "Passive damping of beam vibrations through distributed electric networks and piezoelectric transducers: prototype design and experimental validation," Smart Mater. Struct. 13, pp. 299-308, 2004.

[22] C. Maurini, J. Pouget, and F. dell'Isola, "Extension of the Euler-Bernoulli model of piezoelectric laminates to include 3D effects via a mixed approach," Comput. Struct. 84, pp. 1438-1458, 2006.

[23] B. Lossouarn, M. Aucejo, and J.-F. Deü, "Multimodal coupling of periodic lattices and application to rod vibration damping with a piezoelectric network," Smart Mater. Struct. 24, p. 045018, 2015. 